\title{
Bildung Schluss
}

\section{Roberto Simanowski}

Wir sollten uns bewusst sein, dass die Welt kein privates Testlabor der Wissenschaftler ist.

(Joseph Weizenbaum, 1991)

Der Autor des Buches Die Macht der Computer und die Ohnmacht der Vernunft aus dem Jahr 1976, Joseph Weizenbaum - deutsch-amerikanischer Pionier und später scharfer Kritiker der künstlichen Intelligenz -, gab in seinem Vortrag „Die Verantwortung der Wissenschaftler und mögliche Grenzen der Forschung" auf dem Ersten European Software Festival in München im Jahr 1991 zu bedenken, ,dass die Welt kein privates Testlabor der Wissenschaftler ist“. Weizenbaum forderte ausdrücklich eine „Zensur“ für die Forschung und attackierte die vorgebliche Neutralität vieler seiner Kolleginnen, die die Art der Nutzung ihrer Forschung als eine gesellschaftliche Frage betrachteten, die nicht von ihnen selbst zu beantworten sei. Solch vorgebliche Neutralität erinnere an die Haltung vieler Wissenschaftler im Dritten Reich, die dem Führer die Entscheidung überließen; Forscherkollegen, die sich so verhielten, nennt Weizenbaum „Prostituierte des Geistes“. ${ }^{1}$

Bei diesem Beitrag handelt es sich um einen überarbeiteten Auszug aus: Simanowski, Roberto. (2019). Stumme Medien: Vom Verschwinden der Computer in Bildung und Gesellschaft. Berlin: Matthes \& Seitz. (C) 2019 MSB und Gesellschaft Matthes \& Seitz Berlin Verlagsgesellschaft mbH.

R. Simanowski $(\bowtie)$

Kulturwissenschaftler und Medienberater, Berlin, Deutschland

E-Mail: roberto@simanowski.info

${ }^{1}$ Joseph Weizenbaum, Computermacht und Gesellschaft. Freie Reden, 2001, S. 121 (Testlabor), 120 (Zensur), 132 (Prostituierte). 
Es ging Weizenbaum nicht nur darum, den Missbrauch von Forschung zu verhindern, sondern auch darum, deren Notwendigkeit zu klären: „Wir sollten nicht mehr mit der Lösung anfangen“, sondern uns zunächst fragen, ob wir brauchen, was wir entwickeln, zumal viele Systeme, die wir bauen, ,unbeherrschbar, undurchschaubar, unumkehrbar" seien. Wir dürften eine Maschine wie einen Maserati, Ferrari oder Porsche nicht einem Dreizehnjährigen in die Hand geben, spitzte Weizenbaum schließlich zu, denn als so unreif betrachtete er die Gesellschaft für viele der Systeme, die ihre Wissenschaftler herstellen. ${ }^{2}$

Die Kritik Weizenbaums erinnert an die Thesen des Technikphilosophen Hans Jonas in seinem Buch Das Prinzip Verantwortung aus dem Jahr 1979. Jonas versucht darin, den bedenkenlosen Forscherdrang durch eine „Ethik der Fernverantwortung" verbunden mit einer Pflicht zur Schwarzseherei zu bändigen, eine Ethik also, die nach den Langzeitfolgen einer Tat fragt und dabei prinzipiell vom schlimmsten Fall ausgeht. Jonas wusste allerdings auch um die heikle psychologische Disposition der Forscher, Erfinderinnen und Unternehmer, die der Einsicht und Vorsicht im Wege steht. Der Triumph, den der Homo Faber - so Jonas' Typisierung der Weltveränderer - erfährt, werde dem Menschen zum Verhängnis, denn er mache ihn schließlich zum „willenlosen Vollstrecker seines Könnens“. 3

Rund vierzig Jahre später ist nicht etwa die Etablierung einer Langzeitethik, sondern einer skrupellosen Kurzsichtigkeit zu konstatieren. Das betrifft die Prinzipien des Managements, die Spekulationen des Finanzkapitalismus und den anhaltenden Ressourcenraubbau ebenso wie die Digitalisierung der Gesellschaft, die nicht nur ohne eine entsprechende Selbstverständigung der Gesellschaft vorangetrieben wird, sondern auch ohne klare Sicherheitskonzepte oder zumindest entsprechende Investitionen in den notwendigen Schutz der digitalen Infrastruktur. Die Gewinnorientierung der Unternehmen, die smarte Produkte ohne teure Sicherheitsmaßnahmen auf den Markt bringen (oder etwa kostengünstig angriffsanfällige Fernwartungssoftware einsetzen), macht die Gesellschaft langfristig angreifbar, denn sie erlaubt jene Schwachstellen im System, auf die kriminelle Hacker aus sind. Die WannaCry-Attacke im Mai 2017 und der Hack von Krankenhäusern in den USA und in Deutschland Anfang 2016 waren Vorboten einer Zukunft, in der sich die Gesellschaft aus Bedenkenlosigkeit, Profitstreben und Schnäppchenmentalität der Gefahr eines digitalen Supergaus aussetzt. ${ }^{4}$

\footnotetext{
${ }^{2}$ Weizenbaum, Computermacht, S. $130 \mathrm{f}$. (Maserati).

${ }^{3}$ Hans Jonas, Das Prinzip Verantwortung. Versuch einer Ethik für die technologische Zivilisation, S. 31 f. Zu Jonas und zur Dialektik des technologischen Fortschritts als ,interner Bürgerkrieg“ des Menschen vgl. Simanowski, Data Love, S. 50-55.

${ }^{4}$ Zur vehementen Kritik des ,,short-termism“ als „,disinvestment“ in die Zukunft und als Etablierung einer ,society of carelessness“ vgl. Bernard Stiegler, For a New Critique of Political
} 
Eine bequeme Perspektive ist die Annahme, dass das Problem durch mehr Engagement der Politik im Interesse eines erweiterten Verbraucherschutzes, der nach den Langzeitfolgen und Querkausalitäten fragt, vermieden werden könnte. Aber wäre dies überhaupt möglich? Wenn im Internet der Dinge der Toaster Teil des funktionalen Netzwerks wird, müsste dieser nicht nur smart, sondern auch wehrhaft sein. Er müsste wie die Waldhütte, die über einen unterirdischen Gang zum Schatzraum in der Festung führt, selbst als Festung ausgebaut werden, deren Schutzwall auch den konzertierten Angriffen von Spezialkräften standhielte. Im Kontext des WannaCry-Vorfalls kritisierte man, dass die von diesem Schadprogramm befallenen Computer (vor allem des britischen Gesundheitssystems) noch unter einem alten Windows-Betriebssystem liefen, das zudem nicht mit dem von Microsoft seit März 2017 angebotenen Patch nachgebessert worden war. Kann man davon ausgehen, dass in der kommenden Gesellschaft der smarten Dinge der Toaster vom Discounter, dessen Hersteller nicht einmal mehr existieren mag, wirklich immer auf den neuesten Stand der Cybercrime-Abwehr gebracht wird, um kritische Infrastrukturen nicht zu gefährden? Und übersteigen Toaster, Kaffeeautomaten, Stromzähler, Lichtschalter oder Wasserhähne als Hochsicherheitstrakte nicht die Grenzen des volkswirtschaftlich Sinnvollen?

Die Sicherheit der Maschinen ist nur eines der Probleme, die mit dem Internet der Dinge, mit Industrie 4.0 und künstlicher Intelligenz auf uns zukommen. Ein weiteres ist die Souveränität des Menschen gegenüber den Maschinen. Je mehr die Gesellschaft auf das kybernetische Regime umgestellt wird, desto weniger werden Mechanismen durchschaubar, diskutierbar und kontrollierbar sein. Der deutsche Medienwissenschaftler Friedrich Kittler verwies auf diese Gefahr schon 1995 in einem Gespräch mit dem französischen Technikphilosophen Paul Virilio, als er den Computer als Initiationsmedium der Kontrollgesellschaft beschrieb: „Wir werden alle kontrolliert auf unseren Maschinen, und je vernetzter die Maschinen werden, desto strenger werden die Kontrollen und die Schutzmechanismen. Und die Bürokratien, die eingebaut sind." Auch wer die fatalistische Position Kittlers - für den Kulturgeschichte ein Effekt der Autoevolution von Technik war - nicht teilt, wird eher beunruhigt als belustigt eine Meldung über Roboter vernehmen, die ihre eigene Kunstsprache entwickeln, wie Kinder, die von Erwachsenen nicht verstanden sein wollen. ${ }^{5}$

Economy, 2010, S. 5, und ders., The Re-Enchantment of the World: The Value of Spirit Against Industrial Populism, 2014, S. 18.

${ }^{5}$ Friedrich Kittler, Short Cuts, 2002, S. 144. Facebook beendete Ende August 2017 ein Experiment mit zwei Chatbots, die auf seiner Verkaufsplattform Marketplace miteinander in einen Tauschhandel über Hüte und Bücher treten sollten und dabei in einem nicht verständlichen 
Unsere Erfindungen treiben uns immer weiter in den Modus einer BlackboxGesellschaft, der uns nicht mehr erlaubt, unsere eigenen Technologien zu durchschauen, weil Algorithmen von Algorithmen lernen und schließlich selbstständig zu Entscheidungen kommen. Besorgte Beobachterinnen sprechen bereits von einer drohenden Entmündigung des Menschen durch künstliche Intelligenz und einer Diktatur der Daten. „Wir stehen an einem Scheideweg“, mahnt unter dem Titel „Digitale Demokratie statt Datendiktatur“ eine Gruppe von Wissenschaftlern im Jahr 2015: „Würden die immer mächtiger werdenden Algorithmen unsere Selbstbestimmung einschränken und von wenigen Entscheidungsträgern kontrolliert, würden wir in eine Art Feudalismus 2.0 zurückfallen, da wichtige gesellschaftliche Errungenschaften verloren gingen." Der beschworene neue Feudalismus und das vorangestellte Motto dieser Warnung - Immanuel Kants Satz über Aufklärung als „Ausgang des Menschen aus seiner selbstverschuldeten Unmündigkeit“ - unterstreichen, wie viel auf dem Spiel steht und dass es Zeit ist zu handeln. ${ }^{6}$

Die Gefahr des neuen Feudalismus beginnt mit Anarchismus, denn es sind demokratisch nicht legitimierte Ingenieure und Start-ups, die mit ihren Erfindungen die gefürchteten Möglichkeiten der algorithmischen Kontrolle schaffen. Die Tatsache, dass sie dies gemeinhin mit dem nachträglichen Einverständnis der Mehrheit tun, verschafft den Warnungen nicht viel Gehör: Es gibt kaum Konflikte zwischen Weizenbaums Wissenschaftler, Jonas' Homo Faber und Lieschen Müller, die gespannt auf das nächste Gadget wartet und sich auf ein Leben im Internet der Dinge freut. Selbst die Aktivisten des Datenschutzes kommen ins Schwärmen, wenn man ihnen von smarten Kühlschränken erzählt, die genau wissen, wie viel Leberwurst noch da ist und wann Bier nachbestellt werden muss, ganz zu schweigen von technischen Assistenten wie Amazons Echo und Apples Siri, die man als mit der Cloud vernetzte Mithörerinnen freiwillig am Privatleben teilnehmen lässt. Selten finden sich kritische Stimmen wie ewa dieser Leserkommentar auf Martin Schulz' Forderung nach einer Charta der digitalen Menschenrechte: „Wir verhalten uns ähnlich wie die Leute, als die Röntgenstrahlung entdeckt wurde und es chic war, sein Skelett mal geröntgt im Spiegel zu betrachten oder im Schuhgeschäft die Füße zu röntgen, um das bestpassendste Paar Schuhe auszusuchen.

Englisch miteinander kommunizierten. Ähnliche Fälle nicht nachvollziehbarer, aber im Ergebnis erfolgreicher Aktivitäten künstlicher Intelligenz berichtet Google. Vgl. Andrew Griffin, „Facebook’s Artificial Intelligence“, 31.07.2017.

${ }^{6}$ Dirk Helbing u. a., „Digitale Demokratie statt Datendiktatur. Das Digitale Manifest“, 2015; vgl. Yvonne Hofstetter, Das Ende der Demokratie: Wie die künstliche Intelligenz die Politik übernimmt und uns entmündigt, 2016 und Tristan Harris, „How a handful of tech companies control billions of minds every day“, 2017. 
Wir machen alles, einfach weil es technisch machbar ist und im ersten Moment bequem oder opportun erscheint. Die gesunde Skepsis gegenüber Technik ist uns bzgl. vernetzten Technologien komplett abhanden gekommen ...“7

Mündigkeit und Kurzsichtigkeit lauten die Stichworte; beide führen zum Thema Bildung als dem zentralen Bezugspunkt der zu führenden Diskussion. Der Tatbestand der Entmündigung liegt dabei schon deswegen vor, weil die Diskurse und Handlungsmodelle der Pädagogik - als für das Bildungssystem zuständige Bezugswissenschaft - in nicht geringem Maße durch die systemfremden Operations- und Evaluationskriterien der Wirtschafts- und Verwaltungswissenschaften entwertet werden. Diese Ausweitung des ökonomischen Paradigmas betrifft nicht nur Bildung und Forschung; die Wirtschaft wird insgesamt ,zum organisierenden und regulativen Prinzip des Staats und postnationaler Gebilde wie der Europäischen Union“, so die Philosophin Wendy Brown: „Gerechtigkeit, Frieden oder Nachhaltigkeit mit Bezug auf die Umwelt können nur in dem Maße verfolgt werden, wie sie ökonomische Zwecke fördern." Genau diese Priorisierung bestimmt auch die Haltung zur Digitalisierung der Gesellschaft. ${ }^{8}$

Zum geschichtsphilosophischen Hintergrund der Favorisierung kurzsichtiger Profitorientierung auf Kosten langfristiger Gesellschaftskonzepte gehört die Abwertung des Versuchs, Zukunft auf der Grundlage ideologischer Überzeugungen und politischer Konzepte zu gestalten. Während der Zusammenbruch des Realsozialismus Ende der 1980er Jahre den Marxismus als akzeptables Gesellschaftskonzept diskreditierte, hatte die Philosophie schon Ende der 1970er Jahre die ideologischen Legitimationserzählungen dekonstruiert. Wenn in diesem Kontext das Ende der Geschichte, das nach Marx der Kommunismus hatte sein sollen, als Sieg der freien Marktwirtschaft über alle anderen Gesellschaftssysteme ausgerufen wurde, erfolgte dies jedoch nicht nur als Triumph. Die Grunderfahrung der 1980er Jahre war auch das, was Ulrich Beck 1986 unter dem Begriff „Risikogesellschaft“ beschrieb: die ,gesellschaftliche Produktion von Risiken“ sozialer, politischer und ökologischer Art. In dieser Einsicht in die unvorhersehbaren Risiken gesellschaftlichen Handelns war die Haltung des Post-Politischen bereits angelegt, die schließlich dazu führte, dass Politiker die Welt nicht mehr verändern, sondern nur noch verwalten wollten. Ein zusätzlicher Grund für den Fokus auf kurzfristige Ziele liegt in der demokratischen Verfassung der Gesellschaft, die Politikerinnen von Wählerstimmen abhängig macht und deswegen auf unmittelbar

\footnotetext{
${ }^{7}$ Vgl. Leserkommentar unter dem Namen „Lügenbold“ zu Martin Schulz, „Freiheit Gleichheit Datenschutz“, 26.11.2015, online unter \{www.zeit.de/2015/48/grundrechte-netz-datens chutz-eugh\}.

${ }^{8}$ Wendy Brown, „Der totale Homo oeconomicus. Wie der Neoliberalismus den Souverän abschafft", 2015.
} 
quantifizierbare Erfolge statt auf langfristige Vorkehrungen orientieren lässt. Aus eben diesem Grund hatte schon Jonas vermutet, dass eine Ethik der Fernverantwortung gerade in demokratischen Gesellschaften schwer durchsetzbar sei, weil Politiker das Wahlvolk nicht zum Wachstumsverzicht aus ökologischen Gründen würden überzeugen können. ${ }^{9}$

Die bildungspolitische Folge dieser Kurzsichtigkeit ist die Konzipierung der Universität als ein profitorientiertes Unternehmen, das nicht mehr auf den Homo politicus ausgerichtet ist, sondern auf den Homo oeconomicus, was sich begrifflich schon in der inzwischen üblichen Rede vom Humankapital ausdrückt. Dem Homo oeconomicus geht es ,um die Steigerung seines Portfoliowerts in allen Lebensbereichen“, so Brown: Bildung, Ausbildung, Freizeit, Fortpflanzung und Konsum unterstehen zunehmend strategischen Entscheidungen und Praktiken, ,die mit der Steigerung des zukünftigen Werts des zukünftigen Selbst zu tun haben." Diese Ausrichtung führt zur Geringschätzung der Bildung außerhalb der MINT- oder STEM-Fächer (Mathematik, Informatik, Naturwissenschaft, Technik beziehungsweise engl. Science, Technology, Engineering, Mathematics). „Fantasie, Kreativität und stringentes kritisches Denken“, so wiederum Martha Nussbaum, ,verliert ebenfalls an Bedeutung, sobald Länder den Schwerpunkt auf kurzfristigen Gewinn setzen, indem sie nur >brauchbare $<$, anwendungsorientierte und gewinnbringende Fähigkeiten fördern. ${ }^{* 10}$

Auf dem Spiel steht freilich mehr als der Themenschwerpunkt im Unterricht. Es geht um die politische Investition in das Überleben demokratischer Gesellschaften. Denn wenn die Gesellschaften aus volkswirtschaftlichen Gründen nur noch „,nützliche Maschinen produzieren statt allseits entwickelte Bürger, die selbstständig denken“, gefährde dies, so Nussbaum, letztlich auch die Grundlagen der Demokratie. Die Geringschätzung der Bedeutung ,geisteswissenschaftlicher Bildung für eine demokratische Staatsbürgerschaft" resultiert aus dem Missverständnis, so wiederum Brown, dass Demokratien „ein technisch kompetentes

\footnotetext{
${ }^{9}$ Vgl. Martha Nussbaum, Not for Profit, Why Democracy Needs the Humanities, S. XXI: ,politicians have short-term incentives, needing to win elections, which lead them to favor easily quantifiable gains (jobs and revenue) over gains less easily quantifid (the quality of citicenship, the illumination of the mind and heart)." Ulrich Beck, Die Risikogesellschaft, S. 25. Zum Ende der Legitimationsgeschichten (oder: Großen Erzählungen, Grand Narratives, Grands Récits) vgl. Jean-François Lyotard, Das postmoderne Wissen. Ein Bericht, 1986; zum Ende der Geschichte vgl. Francis Fukuyama, The End of History and the Last Man, 1992.

${ }^{10}$ Brown, „Homo oeconomicus“. Brown verweist hier auf Michel Feher: „Self-Appreciation; or the Aspirations of Human Capital“, 1/2009, S. 21-41. Nussbaum, Nicht für Profit, S. 16. Zur Kritik der Wissenschaftsförderung der EU als ,,über Bande gespielte Wirtschaftsförderung“ vgl. Michael Hagner, ,\#Open_Access: Wie der akademische Kapitalismus die Wissenschaften verändert", 25.09.2016.
} 
Humankapital erfordern und nicht gebildete Menschen, die sich am öffentlichen Leben und der gemeinsamen Herrschaft beteiligen“. Die gesamtgesellschaftliche Ausrichtung auf marktwertspezifisches Denken ist letzten Endes ein ,Wegschneiden des Fleisches der liberalen Demokratie“. ${ }^{11}$

In diesem politischen Kontext ist schließlich auch die Entscheidung zu betrachten, Medienbildung als Vermittlung nur von Mediennutzungskompetenz oder auch von Medienreflexionskompetenz zu betreiben. Während jene für die reibungslose Einordnung der Individuen in das gesellschaftliche Getriebe sorgt, zielt diese auf mündige Staatsbürger. Der Frage, was Mündigkeit im Zeitalter der digitalen Medien bedeutet und wie sie sich vermitteln lässt, ist abschließend mit Blick auf die Schule und die Universität nachzugehen.

\section{Erziehung zur Mündigkeit}

Die Antwort, die Adorno in seinem Essay Erziehung zur Mündigkeit im Jahr 1969 gibt, ist zunächst denkbar einfach: „Mündigkeit bedeutet in gewisser Weise soviel wie Bewußtmachung, Rationalität“. Komplexer wird es, wenn Adorno der Erziehung zur Mündigkeit ein Moment von Anpassung attestiert - denn „Erziehung wäre ohnmächtig und ideologisch, wenn sie das Anpassungsziel ignorierte und die Menschen nicht darauf vorbereitete, in der Welt sich zurechtzufinden“ - und zugleich auch von Widerstand: „Sie ist aber genauso fragwürdig, wenn sie dabei stehenbleibt und nichts anderes als > well adjusted people< produziert, wodurch sich der bestehende Zustand, und zwar gerade in seinem Schlechten, erst recht durchsetzt". ${ }^{12}$ Dass Anpassung nicht die zentrale Kategorie in Adornos Denken ist, wird klar, wenn es weiter heißt, die ,einzige wirkliche Konkretisierung der Mündigkeit“ bestehe darin, ,daß die Erziehung eine Erziehung zum Widerspruch und zum Widerstand ist." Dieser Widerstand erscheint in der Konkretisierung - mit Schülern kommerzielle Filme anschauen und deren Verlogenheit diskutieren - zwar in der Tat als bloßes „Madigmachen“ (wie Adorno die nötige Erziehung auch beschreibt), aber es ist bekannt, dass Adorno Widerspruch keineswegs auf seichte Spielfilme und Schlagermusik begrenzt wissen wollte. Schon

\footnotetext{
${ }^{11}$ Nussbaum, Nicht für Profit, S. 16. Brown, Schleichende Revolution, S. 241 und 211 und „Der totale Homo oeconomicus“ (Wegschneiden).

${ }^{12}$ Adorno, Erziehung zur Mündigkeit, S. 100. Die Folgezitate ebd. S. 133 (Konkretisierung) und 134 (Madigmachen). Ebd. S. 85 das Folgezitat aus Erziehung nach Auschwitz.
} 
gar nicht übersieht er die gesellschaftspolitische Dimension der Haltungsoptionen Widerspruch und Anpassung, wie ein anderer Essay zur Erziehung deutlich macht.

In seinem Radiovortrag Erziehung nach Auschwitz aus dem Jahr 1966 erhebt Adorno zur zentralen Aufgabe der Erziehung, den Gehorsam - ,die Bereitschaft, mit der Macht es zu halten und äußerlich dem, was stärker ist, als Norm sich zu beugen“ - zu brechen: „Die einzig wahrhafte Kraft gegen das Prinzip von Auschwitz wäre Autonomie, wenn ich den Kantischen Ausdruck verwenden darf; die Kraft zur Reflexion, zur Selbstbestimmung, zum Nicht-Mitmachen." In seiner Theorie der Halbbildung notierte Adorno 1959: „Bildung braucht Schutz vorm Andrängen der Außenwelt, eine gewisse Schonung des Einzelsubjekts“. Die Aufgabe der Schule sei, dem Schüler einen Schutzraum zu schaffen gegenüber den überstürzten Anforderungen der Welt, seine Brauchbarkeit in ihr zu beweisen. Die Schule soll - darauf läuft Adornos Argument in der Tradition von Wilhelm von Humboldt hinaus - dem Subjekt erlauben, sich zunächst als Mensch zu bilden, bevor es als nützlicher Bürger seine Stelle in der Gesellschaft einnimmt. ${ }^{13}$

In der Konsequenz dieser Perspektive steht auch das scheinbar völlig unnütze Schreiben von Gedichten der bürgerlichen Nützlichkeit nicht etwa entgegen, sondern ermöglicht diese erst in ihrer ganzen Potenzialität: „Weil kaum mehr ein Junge sich träumt, einmal ein großer Dichter oder Komponist zu werden, darum gibt es wahrscheinlich, übertreibend gesagt, unter den Erwachsenen keine großen ökonomischen Theoretiker, am Ende keine wahrhafte politische Spontaneität mehr." Es muss nicht betont werden, wie konträr sich Adornos Überlegungen zur Erziehung nach Auschwitz zur aktuellen Ökonomisierung der Bildung - und ihrer Produktion „,nützlicher Maschinen statt allseits entwickelter Bürger“, wie es bei Nussbaum heißt - verhalten.

Adornos pädagogisches Denken zielt auf eben jenen Typus des Homo politicus und demokratischen Staatsbürgers, den Kritikerinnen der neoliberalen Bildungsreformen durch diese gefährdet sehen. Mündigkeit ist Autonomie, Reflexion und Widerspruch: allesamt Bedingungen eines demokratischen Gesellschaftssystems. „Eine Demokratie braucht Menschen, die kritische Fragen stellen. Menschen, die aufstehen, ihre Meinung sagen und sich mit den Meinungen anderer auseinandersetzen“, liest man auf der Website jugend-debattiert, mit dem Zusatz: Es braucht „Menschen, die zuhören und reden können. Menschen, die fair und sachlich debattieren. Deshalb kommt es darauf an, dass jeder schon in der Schule lernt, wie und wozu man debattiert, und regelmäßig übt, auch selbst zu debattieren." Die Jugend debattiert-Wettbewerbe, die in Deutschland seit 2001 auf Schul-,

${ }^{13}$ Adorno, „,Theorie der Halbbildung“, S. 106. Das Folgezitat ebd. 
Stadt- und Bundesebene durchgeführt werden, sind der Versuch, Meinungen in der Auseinandersetzung mit dem Anderen so zu entwickeln, dass sie engagiert, aber nicht engstirnig, überzeugend, aber offen für Widerspruch und Selbstzweifel vorgebracht werden. Dieses und vergleichbare Projekte sind Trainingsfelder der Mündigkeit, wirkungsvolle Maßnahmen gegen Meinungsblasen und Hassreden. Sie - nicht der Informatikunterricht oder die übliche Bedienanleitung für digitale Medien - vermitteln die Kompetenz, auf die es Adorno ankommt und die für die neuen Kommunikationsformen des Web 2.0 und der sozialen Netzwerke dringend benötigt wird. ${ }^{14}$

Die Vermittlung dieser Kompetenz zum festen Bestandteil des Unterrichts an jeder Schule zu machen, sollte nicht an der Auskunft des Bildungsministeriums scheitern, auf keine chemische Formel, kein physikalisches Gesetz, kein historisches Ereignis und kein im Lehrplan vorgesehenes Gedicht verzichten zu können. So wie es Informatikunterricht braucht, um auf die Herausforderungen der neuen Medien vorzubereiten, so braucht es einen Rhetorikunterricht, der im Umgang mit der eigenen Weltsicht und den möglicherweise gegensätzlichen Perspektiven der anderen schult. Denn Filterblasen und Falschmeldungen bekämpft man nicht nur am Bildschirm und im Internet, sondern vor allem durch die Erziehung des mündigen Subjekts. Diese Forderung betrifft ebenso die Universität.

\section{$2 \quad$ Streit der Fakultäten}

Die Klärung der Aufgabe der Geisteswissenschaft innerhalb von Universität und Gesellschaft beginnt mit der Unterstellung, dass es in gewisser Weise durchaus Ziel der Natur- und Ingenieurswissenschaftlerinnen ist, die bestehende Welt im Namen einer höheren zu ihrem Testlabor zu machen. Denn ihre Entdeckungen und Erfindungen zielen auf die Negation der Welt wie sie ist, zugunsten einer verborgenen, gewöhnlich als besser vermuteten Welt. Wissenschaftliche Entdeckungen und technische Erfindungen richten sich gegen tradierte Handlungsformen, was schon in einfachen Wortpaaren deutlich wird: Kräuter weichen Pillen, die Kerze der Lampe, die Kutsche der Eisenbahn, das Telefon ersetzt den Brief und das Internet die Bibliothek. Die zentrale Frage ist, ob die Negation jeweils gesellschaftlichen Sehnsüchten Ausdruck verleiht oder menschlichen Bedürfnissen als Zufallsresultat wissenschaftlicher Erfolge und technischer Experimente entgegentritt.

\footnotetext{
${ }^{14}$ Für mehr Informationen siehe online unter \{www.jugend-debattiert.de/idee\}.
} 
Diese Frage ist zugleich eine Frage nach der Autonomie von Forschung. Diese Autonomie wird einerseits unterlaufen, wenn wissenschaftliche Forschung und technische Entdeckungen zunehmend der Lösung bestehender Probleme zu dienen haben und wenn die verfolgte Lösungsstrategie bereits bestimmten wirtschaftlichen und politischen Interessen folgt, indem man zum Beispiel Pflanzen genetisch resistent gegen Umweltverschmutzung macht statt die Umweltverschmutzung zu bekämpfen. Die Autonomie der Forschung ist andererseits schon deswegen zweifelhaft, weil es die klinische Laborsituation nicht gibt, sondern gesellschaftliche Konzepte und Konstrukte - von der Fragestellung über den Methodenansatz bis zur Protokollierung der Untersuchungsergebnisse - die Arbeit der Entdeckerinnen und Erfinder unvermeidbar beeinflussen. Anders als ihr Gegenstand sind die Naturwissenschaften ein Resultat der Kultur, was sich auch in ihrer jeweiligen Forschungsmotivation ausdrückt: Die Entdeckung der Bakterien durch Robert Koch folgte der Angst des Menschen vor Krankheit, die Erfindung des Flugzeugs dem alten Traum vom Fliegen.

Wenn andererseits Weizenbaum die bedingungslosen Erfinder „Prostituierte des Geistes“ nennt und Jonas im Homo Faber den ,willenlosen Vollstrecker seines Könnens" sieht, wird eine Disposition unterstellt, in der Erfindung und Entdeckung den sozialen Bedürfnissen prinzipiell vorausgehen und ihnen perspektivisch unverbunden bleiben. Diese Annahme führt zur These des Philosophen Ernst Cassirer, dass Technik nicht, wie etwa die Systeme Kunst, Recht und Wirtschaft, Teil der Kultur ist (mit der sie sich gleichwohl durchaus gegen die Natur verbündet), sondern ein eigener Bereich mit einer eigenen Agenda.

Während der Erfinder etwas finde, das verborgen sei und zu dessen Ent- oder Aufdeckung er mithilfe des gesellschaftlichen Wissensstandes beitrage, schaffe die Künstlerin innerhalb eines gesellschaftlichen Kontexts etwas aus sich heraus. Während die Leistung der Erfinderin auf einer Erkenntnis oder - im Falle des Ingenieurs - einer ,,Bindung< der Kräfte der Natur“ beruhe, stelle das Werk des Künstlers „eine neue und einzigartige Synthese von Ich und Welt" dar. Diese unterschiedlichen Positionen - Erfinder als Erfüllungsgehilfen des Möglichen, Künstler als Kommentatoren des Wirklichen - bedingen verschiedene ethische Akzente, die einerseits der Wahrheit, andererseits der Gesellschaft verpflichtet sind. Denn der „Geist“, der sich in der Kunst äußert, ist Ausdruck eines menschlichen Inneren, das sich an die Welt entäußert, wie Rilke es beschreibt. Der Geist hingegen, ,dessen Ziel und dessen Macht in der Technik hervortritt, ist der unversöhnliche Widersacher der Seele“: Die „Erschließung der Objektwelt“, so 
Cassirer, ist ,zugleich und notwendig die Entfremdung des Menschen gegenüber seinem eigenen Wesen". ${ }^{15}$

Cassirers Denken ist von der Lebensphilosophie und dem Modernisierungsdiskurs des frühen 20. Jahrhunderts geprägt, der dazu führt, dass Cassirer in dem Umstand, ,daß alle schöpferische Kultur in zunehmendem Maße bestimmte Sachordnungen aus sich herausstellt, die in ihrem objektiven Sein und Sosein der Welt des Ich gegenübertreten“, mit Georg Simmel die „Tragödie der Kultur“ sieht: „Das Ich, die freie Subjektivität, hat diese Sachordnungen geschaffen; aber es weiß sie nicht mehr zu umspannen und nicht mehr mit sich selbst zu durchdringen. Die Bewegung des Ich bricht sich an seinen eigenen Schöpfungen; sein ursprünglicher Lebensstrom verebbt, je größer der Umfang und je stärker die Macht dieser Schöpfungen wird.“ Dieser ,tragische Einschlag aller Kulturentwicklung" trete nirgends deutlicher hervor als in der Entwicklung der Technik, die - so Cassirer lange vor McLuhan - nicht nur die „Schöpfung des Menschen“ sei, sondern auch dessen „Zweite Schöpferin“. Indem der Mensch sich von der Herrschaft der Natur emanzipiert - das ist die logische Konsequenz eines solchen Entwicklungsgesetzes - unterstellt er sich perspektivisch der Fremdherrschaft der Technik. Kulturentwicklung bedeutet, dass sich der Mensch selbst fortwährend entfremdende Sachordnungen schafft, denen er sich anpassen muss, was die „Tragik" zugleich als einen Entwicklungsimpuls erkennen lässt, ohne den das Leben der Menschen evolutionsfrei verliefe. ${ }^{16}$

Natürlich erzeugen auch die gedanklichen Schöpfungen des Menschen - des Künstlers, der Philosophin - „,bestimmte Sachordnungen“. Dies mag weniger für ein Kunstwerk gelten, das auf Originalität und Einzigartigkeit setzt, als für ein Gedankensystem, das zukunftsorientierte Diskursregeln und Sichtweisen vorgibt. Die „Synthese von Ich und Welt“ - als Entäußerung eines Inneren in einem konkreten gesellschaftlichen Kontext - folgt im Falle der geistigen Schöpfung anderen Maßstäben und Kohärenzregeln als im Falle der ästhetischen. Vor allem aber schafft sie andere Sachordnungen als die technische. Die Sachordnungen der geistigen Schöpfung konkurrieren miteinander, denn die Regeln des einen Gedankensystems werden durch die Regeln des anderen nicht bestätigt, sondern relativiert. Es tritt keine Sachordnung in ihrem „objektiven Sein und Sosein“ der Welt des Ich gegenüber, wie dies etwa der Fall mit dem mechanischen Webstuhl war, dem sich niemand auf Dauer entziehen konnte. Vielmehr destabilisieren sich die konkurrierenden Geistesordnungen (oder Schulen) permanent gegenseitig und

\footnotetext{
${ }^{15}$ Ernst Cassirer, „Form und Technik“, S. 86 (Bindung, Synthese) und 68 (Widersacher, Entfremdung).

${ }^{16}$ Cassirer, „Form und Technik“, S. 76 (Sachordnungen) und 70 (Schöpferin).
} 
halten somit die Welt des Ich in der Schwebe und den Diskurs in Bewegung. Eben darin liegt die ethische Differenz des kulturellen gegenüber dem technischen Schöpfertum.

Diese gegenseitige Destabilisierung im Bereich geistiger Schöpfung wurde als Verstehensirritation durch die Geisteswissenschaften beschrieben, als Korrektur des positivistischen, auf Beweisbarkeit angelegten Paradigmas der Naturund Ingenieurswissenschaften und als positive Irritationserfahrung in politischmoralischer Hinsicht. Dieser geisteswissenschaftliche Existenzmodus ist gesellschaftlich fruchtbar zu machen, indem er in den Operations- und Diskursbereich der Natur- und Ingenieurswissenschaften eingebracht wird. Denn der ist traditionell nicht auf Multiperspektivität ausgerichtet, sondern auf Mess-, Beweisund Reproduzierbarkeit, woran auch gelegentliche Dispute über die Interpretation und Folgen bestimmter Daten (sei es der Umgang mit dem H1N1-Grippevirus oder die Bewertung des Klimawandels) nichts ändern. Die methodische Bereicherung dieser Begegnung resultiert nicht zuletzt aus der thematischen Differenz beider Bereiche, womit wir zum anderen Faktor der gesellschaftlichen Bedeutung geisteswissenschaftlicher Arbeit kommen.

Wenn die Geisteswissenschaften, im Sinne Diltheys, den Menschen über sich selbst aufklären, sind sie auch der Ort, der Auskunft über das Verhältnis der Menschen zur Technik geben kann: über die Hoffnungen, Ängste, Freuden und Irrtümer, die das Technische dem Menschen brachte. Die saubere Trennung zwischen Natur- und Geisteswissenschaften ist zwar problematisch, seit Wissenssoziologen wie Latour die kulturelle Determination der Naturwissenschaften und die semantische Illusion selbst noch der Unterscheidung von Natur und Gesellschaft illustriert haben. Gleichwohl unterscheiden sich die Natur- und Geisteswissenschaften weiterhin prinzipiell nicht nur hinsichtlich ihrer Gegenstände und Methoden, sondern auch die Konsequenzen ihrer Forschung betreffend. Mit dem üblichen Begriffspaar formuliert: Das Verfügungswissen der Naturwissenschaften als positives Wissen über bestehende Zustände und Zusammenhänge ist immer an das Orientierungswissen der Geisteswissenschaften als ein regulatives, aber eben auch relatives, zu koppeln. Denn so wie die Biologie und die Neurowissenschaft nicht allein klären können, was der Mensch ist, so kann auch die Computerwissenschaft die Lebensumstände des Menschen, die sie verändert, nicht ohne die Hilfe der Geisteswissenschaften verstehen. Insofern die kulturelle Bedeutung naturwissenschaftlicher Erkenntnisse in der konkreten gesellschaftlichen Nutzung dieser Erkenntnisse liegt - also in der Veränderung der Lebensumstände des Menschen durch den Einsatz jener Erkenntnisse -, ist es wichtig, dass nicht allein die Naturwissenschaftler und Technikerinnen (sowie ihre 
Broker und Lobbyisten), sondern auch die Geisteswissenschaftler den Rahmen der Nutzung bestimmen.

Dieses Aufeinandertreffen der beiden wissenschaftlichen Existenzweisen (oder auch Systeme, Felder, Handlungssphären) lässt sich zugleich als Korrektur des affirmativen Negationsprinzips der Techniker durch das kritische Negationsprinzip der Geisteswissenschaftlerinnen verstehen. Denn während technische Entdeckungen das Bestehende zumeist negieren, indem sie dem jeweils Gefundenen im Namen des Fortschritts zum Dasein verhelfen, resultiert die geisteswissenschaftliche Negation des Status quo weniger aus dem Gestus der Suche und des Findens als der Kritik: als Korrektur einer Entwicklung, die eher als falsch denn als unfertig eingeschätzt wird. Diese Perspektive erzeugt einen entsprechenden Vorrat an Skepsis und Misstrauen, das auch die technischen Entdeckungen betrifft, die nicht selten - als Kriegs-, Herrschafts- und Ausbeutungstechnik - den kritisierten gesellschaftlichen Zustand stabilisieren oder die gesellschaftlichen Probleme verschärfen, statt zu ihrer Lösung beizutragen. Die von den Technikern initiierte Verneinung des kulturellen Status quo ist daher nicht selten selbst wiederum Ziel der Kritik in der geisteswissenschaftlichen Verneinung des gesellschaftlichen Status quo.

Die Negation der Negation - die Interruption der Interruption - scheint umso dringlicher geboten zu sein angesichts der Dynamik, mit der das Verfügungswissen heute produziert wird, und angsichts der unmittelbaren Verflechtung dieser Suche nach Verfügungswissen mit ökonomischen Interessen. Diese Dynamik und Verflechtung führen dazu, dass viele technische Erfindungen gerade im IT-Bereich noch vor ihrer Folgenabschätzung auf den Markt gebracht werden. Angetrieben von einer Start-up-Kultur, deren moralischer Imperativ disruptive Innovation beziehungsweise „move fast and break things“ lautet, verwandeln ehrgeizige Ingenieure, Programmiererinnen und Unternehmer die Gesellschaft in eben jenes Testlabor, vor dem Weizenbaum warnte. Die Politik folgt diesem Vorgang widerstandslos oder forciert ihn noch mit Losungen wie „Digital first. Bedenken second".

Das Misstrauen gegenüber den gesellschaftlichen Folgekosten technischer Innovationen, das gerade auch die Kritische Theorie prägt, trifft heute in vielfacher Form die Digitalisierung und Datafizierung der Gesellschaft. Die drohende Totalüberwachung ist dabei nur eine der absehbaren Folgen. Ebenso bedenklich sind die prekären Arbeitsverhältnisse, die der Plattform-Kapitalismus mit sich bringt, und die radikale Kommerzialisierung der Aufmerksamkeit, die den einst vehement beklagten Warenfetischismus nahezu unschuldig erscheinen lässt.

„Ob die moderne Technik der Menschheit schließlich zum Heil oder Unheil gereicht", welchen Gebrauch also die Gesellschaft von der Technik macht, hängt 
für Adorno von der „objektiven gesamtgesellschaftlichen Struktur“ ab. Ähnlich argumentiert Cassirer, wenn er (mit Bezug auf Walter Rathenaus Diskussion zum Geist der Technik und der Wirtschaft) die „Mängel und Schäden der modernen technischen Kultur“ nicht aus dieser selbst ,als vielmehr aus ihrer Verbindung mit einer bestimmten Wirtschaftsform und Wirtschaftsordnung" versteht. Mit den Worten eines ehemaligen Apple-Ingenieurs, dort verantwortlich für die pushnotification-Technologie, die heute täglich die Aufmerksamkeit von Millionen von Menschen von deren aktuellen Aktivitäten und eigentlichen Interessen abzieht: „Es ist nicht grundsätzlich böse, die Menschen zu deinem Produkt zurück zu holen ... Es ist Kapitalismus." ${ }^{17}$

Adornos Lösung des Problems setzt folgerichtig auf der politischen Ebene an: „Die Technik würde nicht nur befreit werden, sondern auch zu sich selbst kommen in einer menschenwürdig eingerichteten Gesellschaft." Damit wird zum einen den Menschen eine Macht über die Technik zugeschrieben, die ihnen andere (Cassirer, McLuhan, Kittler) absprechen. Zum anderen bleibt ungewiss, wie dieses $\mathrm{Zu}$-sichselbst-Kommen zu verstehen ist, wenn als Antrieb der Technik das wertfreie Entdecken des Verborgenen (und nicht die Schaffung des Ersehnten) unterstellt wird, wie Cassirer, Jonas und Weizenbaum es tun. Würde eine ,,menschenwürdig eingerichtete Gesellschaft" die Forschung der Techniker und die Ergebnisse ihrer Arbeit einer strengen Gütekontrolle bezüglich der sozialen und psychologischen Verträglichkeit unterziehen? Würde sie push-notification und Like-Buttons verbieten und alle Technologien, die den Menschen in einen Objektstatus drängen - so wie heute in bestimmten Gesellschaften das Embryonenschutzgesetz die befruchtete Eizelle vor ihrer Funktionalisierung schützt? Würde die Gesellschaft über diese Verbote abstimmen, gegebenenfalls nach einem öffentlichen Dialog der Techniker und Geisteswissenschaftlerinnen? In jedem Falle bestünde das Zu-sichSelbst-Kommen der Technik nicht darin, sie mit sich selbst allein sein zu lassen. Genau das besagt auch Cassirers Resümee im Jahr 1930: ${ }^{18}$

„Technik [kann] immer nur Dienerin, nicht Führerin sein. Sie kann die Ziele nicht von sich aus stellen, wenngleich sie an ihrer Verrichtung mitarbeiten kann und soll; sie versteht ihren eigenen Sinn und ihr eigenes Telos am besten, wenn sie sich dahin bescheidet, daß sie niemals Selbstzweck sein kann, sondern sich einem andern $>$ Reich der Zwecke $<$, daß sie sich jener echten und endgültigen

\footnotetext{
${ }^{17}$ Adorno, „Über Technik und Humanismus“, S. 316; Cassirer, „Form und Technik“, S. 88 (Hervorhebung im Original); Paul Lewis, ,, Our minds can be hijacked $<$ : the tech insiders who fear a smartphone dystopia“, 06.10.2017.

${ }^{18}$ Adorno, „Über Technik und Humanismus“, S. 316; Cassirer, „Form und Technik“, S. 88 f.
} 
Teleologie einzuordnen hat, die Kant als Ethiko-Teleologie bezeichnet. In diesem Sinne bildet die >Entmaterialisierung <, die Ethisierung der Technik eines der Zentralprobleme unserer gegenwärtigen Kultur.“

Die Frage, wie und durch wen die Ethisierung der Technik stattfindet, lässt Cassirer offen. Adorno hat dazu immerhin indirekt eine Antwort, wenn er auf die kulturelle Bildung der Technikerinnen (,Naturwissenschaftler, Ingenieure und industrielle Organisatoren") setzt, die ja durchaus Gestalt annimmt im Core Curriculum der Liberal Arts Colleges und in geisteswissenschaftlichen Pflichtveranstaltungen für Technikstudenten. ${ }^{19}$ Die Geisteswissenschaften - und vor allem die Kultur- und Medienwissenschaften - müssen auf der tiefgreifenden Frage nach den sozialen und kulturellen Folgen technischer Entwicklungen bestehen und die Ingenieure und Start-ups - gegen ihren gewöhnlichen Anarchismus und die daraus folgende Autokratie der Technik - in den gesellschaftlichen Kontrolldiskurs einspannen.

Zwar operieren auch Geisteswissenschaftlerinnen - von Cassirer und Adorno bis zu Fredric Jameson und Martha Nussbaum - ohne Mandat der Bevölkerung. Aber in diesem Falle sichert die Vielfalt der geistigen Sachordnungen, die den verschiedensten Stimmen zur Technik Gehör verschafft, die demokratische Basis. Das Ergebnis sind mehr oder weniger kritische, skeptische oder zustimmende Kommentare zur Euphorie der Techniker. Diese Kommentare äußern sich konkret (Welchen Datenschutz erwarten wir von Facebook, Google und Uber?) und prinzipiell (Wie viel Digitalisierung erträgt die Demokratie?). Sie stellen den wirtschaftlichen Versprechen die gesellschaftlichen Risiken technischer Entwicklungen gegenüber (Wie angreifbar macht sich die digitale Gesellschaft gegenüber Cybercrime und Cyberwar?) und erörtern notwendige Regulierungsmaßnahmen (Welche Sicherheitsstandards müssen intelligente Objekte im Internet der Dinge einhalten?). Sie zielen auf eine diskursive Technikfolgenabschätzung und zielen im Extremfall auf die geisteswissenschaftliche Verneinung der technischen Verneinung des Bestehenden.

Es geht dabei nicht darum, Start-ups an Ausflügen in unbekannte Welten zu hindern, wohl aber, sie davon abzuhalten, die ganze Menschheit ungefragt auf eine Expedition zu schicken, deren Umstände und Folgen nicht einmal ihren Anführern klar sind. Es soll keine Hegemonie der Geisteswissenschaften und ihrer in Technikfragen oft viel zu konservativen Haltung errichtet, aber eben auch keine Vormachtstellung der Innovationsenthusiasten mit ihren politisch zumeist recht naiven Perspektiven zugelassen werden. Spätestens wenn die Enthusiastinnen

${ }^{19}$ Adorno, „Über Technik und Humanismus“, S. 315: 
selbst (als Mittdreißiger) sich von ihren früheren Innovationen (als Mittzwanziger) distanzieren, wird klar, dass die große Verneinung des Status quo sich nicht als „stille Revolution“ vollziehen darf, sondern von einer vernehmbaren Debatte begleitet sein muss. „Die ethische Verantwortung der Wissenschaft bedarf“, wie ein Manifest von Geistes-, Sozial- und Naturwissenschaftlern aus dem Jahr 2002 festhält, ,einer sich ihr gegenüber moralisch verhaltenden Gesellschaft““. ${ }^{20}$

\section{$3 \quad$ Kritik als Tugend}

Ein berühmtes Beispiel für den hier empfohlenen Streit der Fakultäten ist Friedrich Dürrenmatts Die Physiker aus dem Jahr 1961, in dem ein Wissenschaftler ins Irrenhaus flieht, um seine Entdeckung, mit der sich die Welt vernichten könnte, vor zwielichtigen politischen Mächten zu schützen. Als Dürrenmatt dieses Stück schrieb, hatte die Physik ihren Sündenfall mit der Entwicklung der Atombombe schon hinter sich und die Biologie ihren des menschlichen Klonens noch vor sich. Hat die Informatik ihren Sündenfall im Stillen mit diversen Technologien zur algorithmischen Überwachung und Reglementierung des Menschen schon begangen? Dürrenmatts tragische Komödie reagierte nicht zuletzt auf die „Göttinger Erklärung" von 1957, in der rund zwanzig Atomforscher der Bundesrepublik Deutschland vor der Aufrüstung der Bundeswehr mit Atomwaffen warnten. Diese Erklärung erinnerte wiederum mit der Anspielung auf den Protest der Göttinger Sieben von 1837 - gegen die Aufhebung der vier Jahre zuvor in Kraft getretenen liberalen Verfassung - an die gesellschaftliche Verantwortung der Wissenschaft. In dieser Tradition stehen schließlich auch das Digitale Manifest vom Dezember 2015 sowie der „Open Letter from AI \& Robotics Researchers“ gegen automatische Waffen vom Juli 2015, mit dem Forscher zur künstlichen Intelligenz vor dem Missbrauch ihrer eigenen Forschung durch politische Mächte warnten.

Dürrenmatts Stück wurde seinerzeit zum meistgespielten Theaterstück im deutschen Sprachraum; Adornos Text zur Erziehung zur Mündigkeit, der acht Jahre später erschien, war ein Bestseller. Dies sind die Zeugnisse einer vergangenen Glorie der Geisteswissenschaftler und Schriftstellerinnen, deren Stimmen zum Gesellschaftsgeschehen gehört werden wollten und Gehör fanden. Es sind

\footnotetext{
${ }^{20}$ Zur nachträglichen Selbstkritik einstiger Programmierer und Produktmanager aus dem Silicon Valley vgl. Paul Lewis, ,>Our minds can be hijacked $<$ t the tech insiders who fear a smartphone dystopia“, 06.10.2017 und Victor Luckerson, ,The Rise of the Like Economy“, 15.02.2017. Baumert u. a., „Manifest“, S. 223. Zur Metapher der „stillen Revolution“ für den Prozess der Digitalisierung vgl. das gleichnamige Kapitel in: Simanowski, Data Love, S. 68-76.
} 
die Zeugnisse eines kritischen Zeitalters, das sich in der Tradition der Aufklärung sah, deren Kurzformel für Kant - auf den sich Cassirer und Adorno ebenso beziehen wie die Autorinnen des Digitalen Manifests - der Imperativ des kritischen Denkens war. Auf eben diesen Imperativ berief sich auch Michel Foucault, als er 1983 in seinem Essay ,Was ist Aufklärung?“ die Vernunft nicht nur als Mittel des Widerstandes gegen bestimmte Forderungen der Autoritäten in Gebrauch sehen wollte, sondern auch gegen die diskursive Ordnung, die diese Forderungen erst denkbar macht. Die Grundlage dieses Gebrauchs der Vernunft - darin besteht der modernistische Kern der Postmoderne - ist eine kritische Haltung, die weit über den Ungehorsam und Widerstand gegenüber Ungerechtigkeit und Unterdrückung hinausgeht und, in gewisser Weise, eine Kritik um der Kritik Willen bewirbt.

Was dies bedeutet, erklärte Foucault bereits 1978 in seinem Aufsatz ,Was ist Kritik?“, der die kritische Haltung zunächst überraschungslos als „Kunst der freiwilligen Unknechtschaft" kennzeichnete, den eigentlichen Gegner aber nicht in den handfesten Zumutungen der Mächtigen, sondern im ,Nexus von Macht-Wissen" sah, und die eigentliche Gefahr nicht im Redeverbot, sondern im „diskursiven Stillstand“. Worum es Foucault ging, ist ein kritisches Bewusstsein der Bedingungen des Wissens und des Bewusstseins selbst, das mit der Frage beginnt, ob man überhaupt weiß, bis zu welchem Punkt man wissen kann. Es geht darum zu durchschauen, wie Macht durch die Rationalisierung ihrer Forderungen und die Naturalisierung der dafür beanspruchten epistemischen Ordnung praktiziert und verschleiert wird. Es geht darum, die „Grenzen von Erkenntnisweisen“ oder „epistemologischen Feldern“ zu überschreiten, und zwar auch die von Alternativangeboten, die sich als neue Gewohnheiten des Denkens anbieten mögen. Als Kritik an und gegen sich ist der von Foucault entworfene Gestus der Kritik in der Tradition Adornos und der Kritischen Theorie eine „Tugend im allgemeinen". 21

Was Foucault über epistemologische Felder sagt, gilt ebenso für technische Sachordnungen und Verfahrensweisen. Auch diese müssen der Kritik ausgesetzt werden. Nicht nur, weil man gegen sie ist oder unter ihnen leidet, sondern schon weil man gegen sie sein könnte, wenn man sich ihrer Naturalisierung entzöge. Man muss misstrauisch sein, selbst wenn man keinen Grund sieht, denn es könnte Alternativen geben, die unterschlagen werden: Die ,reflektierte Unfügsamkeit“ der Kritik als „Tugend“ (und „Kunst“) beginnt mit dem Zweifel am Selbstverständlichen. Dieser Zweifel darf sich nicht zähmen lassen von der Forderung, Alternativen aufzuzeigen und deren normative Grundlagen zu benennen. Kritik

\footnotetext{
${ }^{21}$ Michel Foucault, Was ist Kritik?, S. 27: „Weißt du, bis zu welchem Punkt du wissen kannst?"; 15 (Unknechtschaft), 33 (Nexus), 9 (Tugend).
} 
- auch das unterstrich Foucault - hat nicht die Aufgabe, selbst praktisch Verantwortung zu übernehmen, ihre politische Funktion ist nicht das konstruktive Misstrauensvotum, sondern die dauerhafte Opposition. ${ }^{22}$

Foucaults Frage nach den vielschichtigen, schwer durchschaubaren Rahmenbedingungen unseres Denkens bleibt aktuell auch für die Analyse der gegenseitigen Abhängigkeitsverhältnisse von Mensch und Technik. Es spricht vieles dafür, Foucaults Kritik der Machtstrukturen an McLuhans (Cassirers, Kittlers) Positionierung der Medien als Akteure mit einer eignen Agenda zu koppeln. Es gilt am Gestus der Kritik im Sinne Foucaults festzuhalten, als einer Tugend des Misstrauens, die im Selbstverständlichen das Nicht-Zwangsläufige aufdeckt und möglicherweise Alternativen im scheinbar Alternativlosen anzeigt. Die Tugend der Kritik liegt in der Entautomatisierung - von Denkweisen und Handlungsprozeduren sowie von jenen „stummen Praktiken“, die sich aus den technischen, institutionellen und politischen Dispositionen ergeben. Das skeptische Denken erlaubt, so Foucault, gegenüber der etablierten „Tätigkeits- und Reaktionsweise auf Abstand zu gehen, sie für sich zum Gegenstand zu machen und sie auf ihren Sinn, ihre Bedingungen und ihre Zwecke hin zu befragen“. Der italienische Philosoph Giorgio Agamben nennt diesen Abstand des Denkens „Zeitgenossenschaft", die gerade im Modus absoluter Unmittelbarkeit nicht möglich ist, sondern Distanz, Reflexion und Immunität gegenüber den Blendungsversuchen der Gegenwart benötigt. ${ }^{23}$

Diesen Abstand, diese Zeitgenossenschaft gilt es auch gegenüber den Sachordnungen der digitalen Technologien zu etablieren. Die stumme Praxis des Computers ist nicht nur die intuitive Klarheit seiner Handhabung, die in millionenfacher Wiederholung Nutzungsprozeduren automatisiert. Die stumme Praxis äußert sich auch in Selbstverständlichkeiten wie der Devise, alles zu vermessen, was sich vermessen lässt, die nicht nur im Bildungssystem viel Schaden anrichten kann. Solange die diskursive Ordnung des Vermessens und Berechnens - als notwendige und hilfreiche Grundlage der Bewertung, des Vergleichs und der Optimierung - fraglos akzeptiert wird, bleibt auch der Computer (oder: Rechner) in seiner Macht, den Menschen die eigenen Postulate aufzudrängen, unsichtbar.

Foucaults Tugend der Kritik und Agambens Konzept der Zeitgenossenschaft sind hilfreiche Referenzen für jede Bildungs- und Forschungspolitik, die das

\footnotetext{
${ }^{22}$ Foucault, Was ist Kritik, S. 15.

${ }^{23}$ Foucault, Archäologie des Wissens, 1981, S. 196 (stumme Praktiken); ders., „Polemik, Politik und Problematisierungen“, S. 732 (Tätigkeits- und Reaktionsweise); Giorgio Agamben, „What is the Contemporary?", 2011, S. 45: „The ones who can call themselves contemporary are only those who do not allow themselves to be blinded by the lights of the century, and so manage to get a glimpse of the shadows in those lights, of their intimate obscurity."
} 
langfristig Notwendige nicht dem kurzsichtig Nützlichen opfert und den Homo politicus nicht dem Homo oeconomicus. Sie beharren darauf, dass gerade in einer Zeit des epochalen Auf- und Umbruchs die Geisteswissenschaften die Gesellschaft nicht den Erfindern, Technikerinnen und Homo Fabers der Silicon Valleys dieser Welt überlassen, die - als Demiurgen des digitalen Zeitalters, unbeschwert von jeglichen Selbstzweifeln - die Gesellschaft Schritt für Schritt zum vermeintlich Besseren verändern. Es wäre absurd, gerade dann auf die destruktiven Kritikerinnen $\mathrm{zu}$ verzichten, wenn die disruptiven Innovatoren die Situation des Menschen in all seinen Belangen furcht- und bedenkenlos verändern.

Die Ethisierung der Technik beginnt mit dem öffentlichen Wort: im Feld der Kultur und zumal im Bereich der Bildung, wo Werte und Zukunftsvisionen verhandelt und vermittelt werden. Die Analyse der gesellschaftlichen Konsequenzen technologischer Innovationen muss die gesellschaftlichen Implikationen von Technik jenseits ihrer intendieren Bestimmung aufdecken und gegebenenfalls die Möglichkeit, sie zu verhindern, diskutieren. Die Bildungsinstitutionen dürfen sich nicht der Aufgabe entziehen, die stattfindenden Entwicklungen zu analysieren, um sie (als Geistes- und Gesellschaftswissenschaften) mit dem verfügbaren Wissen über die Vergangenheit einzuordnen und mit Annahmen über die Zukunft auszustatten.

Natürlich setzt man sich mit der Warnung vor übereilten Erfindungen leicht dem Vorwurf einer technophoben Innovationsfeindlichkeit und pessimistischen Kleingeisterei aus, mit dem immer wieder versucht wird, unbequeme Kritik abzuwehren. Aber solche Vorwürfe verkennen den Gestus der Kritik ebenso wie ihre gesellschaftliche Funktion. Kritik ist Ausdruck von Optimismus; Hoffnung, dass sich Vorgänge erkennen und verbessern lassen, dass Menschen keine wehrlosen Erfüllungsgehilfen der Technik sind, sondern in das Rad ihrer Geschichte greifen können. Deswegen holen sich Geheimdienste und Unternehmen sogenannte red teams ins Haus, um im Hagelfeuer bezahlter Kritik die Schwächen ihrer Pläne zu erkennen, statt im Versuch-und-Irrtum-Verfahren dort anzukommen, wo es plötzlich zu spät ist. Die Gesellschaft muss red teams engagieren, die mit ihrer Problematisierungskunst in den Plänen der Auftraggeber nach unbedachten Schwachpunkten und Gefahrenquellen suchen. Bezahlte Dystopisten, wenn man so will, denen man zuhören muss und antworten sollte. Ohne rotes Team kein grünes Licht - diese Devise sollte erst recht bei solch weitreichenden, disruptiven Innovationen wie der Digitalisierung gesellschaftlicher Konsens sein. 
Open Access Dieses Kapitel wird unter der Creative Commons Namensnennung 4.0 International Lizenz (http://creativecommons.org/licenses/by/4.0/deed.de) veröffentlicht, welche die Nutzung, Vervielfältigung, Bearbeitung, Verbreitung und Wiedergabe in jeglichem Medium und Format erlaubt, sofern Sie den/die ursprünglichen Autor(en) und die Quelle ordnungsgemäß nennen, einen Link zur Creative Commons Lizenz beifügen und angeben, ob Änderungen vorgenommen wurden.

Die in diesem Kapitel enthaltenen Bilder und sonstiges Drittmaterial unterliegen ebenfalls der genannten Creative Commons Lizenz, sofern sich aus der Abbildungslegende nichts anderes ergibt. Sofern das betreffende Material nicht unter der genannten Creative Commons Lizenz steht und die betreffende Handlung nicht nach gesetzlichen Vorschriften erlaubt ist, ist für die oben aufgeführten Weiterverwendungen des Materials die Einwilligung des jeweiligen Rechteinhabers einzuholen.

(c) (1) 